PROCEEDINGS OF THE AMERICAN MATHEMATICAL SOCIETY

Volume 124, Number 11, November 1996

\title{
GENERALIZED CUNTZ-KRIEGER ALGEBRAS
}

\author{
VALENTIN DEACONU
}

(Communicated by Palle E. T. Jorgensen)

\begin{abstract}
To a special embedding $\Phi$ of circle algebras having the same spectrum, we associate an r-discrete, locally compact groupoid, similar to the Cuntz-Krieger groupoid. Its $\mathbf{C}^{*}$-algebra, denoted $\mathcal{O}_{\Phi}$, is a continuous version of the Cuntz-Krieger algebras $\mathcal{O}_{A}$. The algebra $\mathcal{O}_{\Phi}$ is generated by an AT-algebra and a nonunitary isometry. We compute its K-theory under the assumption that the AT-algebra is simple.
\end{abstract}

\section{INTRODUCTION}

The idea of considering continuous versions of the Cuntz-Krieger algebras $\mathcal{O}_{A}$ occured to us as a result of studying the groupoid approach to the Cuntz algebra $\mathcal{O}_{n}$. In [De1], we treated continuous versions of $\mathcal{O}_{n}$, replacing the Cantor set and the unilateral shift by a compact space and a self-covering. In this paper we are concerned with a local homeomorphism $\sigma$ on a space $X$ of infinite paths in a diagram arising from an embedding $\Phi$ of circle algebras. We suppose that the circle algebras have the same spectrum; therefore the infinite stationary diagram associated with the embedding has a vertical symmetry, and it carries a Markov shift, denoted by $\sigma$. We study the $\mathbf{C}^{*}$-algebra $\mathcal{O}_{\Phi}$ of a groupoid constructed from the pair $(X, \sigma)$, in analogy with the Cuntz-Krieger groupoid. This algebra has a structure which is similar to that of the algebras $\mathcal{O}_{A}$ : it is a crossed product of an inductive limit by an endomorphism. However here, instead of an AF-algebra, we have an AT-algebra.

Our main result is that under natural conditions that imply the simplicity of the AT-algebra, we have

$$
\begin{aligned}
& K_{0}\left(\mathcal{O}_{\Phi}\right) \simeq \operatorname{ker}\left(i d-\Phi_{1}\right) \oplus\left(\mathbf{Z}^{N} /\left(i d-\Phi_{0}\right) \mathbf{Z}^{N}\right), \\
& K_{1}\left(\mathcal{O}_{\Phi}\right) \simeq \operatorname{ker}\left(i d-\Phi_{0}\right) \oplus\left(\mathbf{Z}^{N} /\left(i d-\Phi_{1}\right) \mathbf{Z}^{N}\right) .
\end{aligned}
$$

Here $\Phi_{*}=K_{*}(\Phi): \mathbf{Z}^{N} \rightarrow \mathbf{Z}^{N}$ are the matrices induced by $\Phi$ at the level of $\mathrm{K}$ theory. After a first version of this result was included in my thesis, I learned from M. Dădârlat that recent results of M. Rørdam prove that the simple infinite algebras $\mathcal{O}_{\Phi}$ can be classified by their K-theory groups (see $[\mathrm{R} \varnothing]$ ).

It should be mentioned that recent investigations of I. Putnam and M. Pimsner also treat generalizations of the Cuntz-Krieger algebras, using different methods.

Received by the editors May 15, 1995.

1991 Mathematics Subject Classification. Primary 46L05, 46L55, 46L80.

(C)1996 American Mathematical Society 


\section{Definition of $\mathcal{O}_{\Phi}$}

In the case of Cuntz-Krieger algebras $\mathcal{O}_{A}$, the starting point is an $N \times N, 0-1$ matrix $A$. This matrix (or rather its transpose) describes an inclusion of finite dimensional algebras with the same spectrum ( $N$ points),

$$
A^{t}: \bigoplus_{k=1}^{N} \mathbf{C} \rightarrow \bigoplus_{k=1}^{N} \mathbf{M}_{p_{k}},
$$

by means of a Bratteli diagram. Here $p_{k}=\sum_{i=1}^{N} A_{i k}$, and we put an edge from the vertex $i$ to the vertex $j$ iff $A_{i j}=1$. For example, in Figure 1, we have the diagram corresponding to

$$
A=\left(\begin{array}{lll}
1 & 1 & 0 \\
1 & 0 & 1 \\
1 & 0 & 0
\end{array}\right)
$$

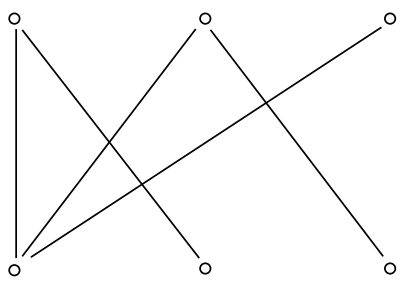

Figure 1

The main idea in the generalization is to use disjoint unions of circles as vertex spaces and to consider edges which will describe a 'nice' inclusion

$$
\Phi: \bigoplus_{k=1}^{N} C(\mathbf{T}) \rightarrow \bigoplus_{k=1}^{N} C(\mathbf{T}) \otimes \mathbf{M}_{p_{k}} .
$$

The building blocks of the homomorphism $\Phi$ we take to be $\sigma_{p}$ and $\hat{\sigma}_{q}$, where $p, q$ are non-zero integers, and where

$$
\sigma_{p}: C(\mathbf{T}) \rightarrow C(\mathbf{T}), \quad\left(\sigma_{p} f\right)(z)=f\left(z^{p}\right),
$$

and

$$
\hat{\sigma}_{q}: C(\mathbf{T}) \rightarrow C(\mathbf{T}) \otimes \mathbf{M}_{|q|},
$$

taking the generator $z$ into

$$
\left(\begin{array}{cccc}
0 & \ldots & 0 & z \\
1 & \ldots & 0 & 0 \\
\vdots & \ddots & \vdots & \vdots \\
0 & \ldots & 1 & 0
\end{array}\right), \text { if } q>0
$$

with $z$ replaced by $\bar{z}$ if $q<0$. The map $\hat{\sigma}_{q}$ is also called the $q$-times around embedding, or the homomorphism compatible with the covering $z \mapsto z^{q}$. These building blocks are described by the diagrams in Figure 2.

Note the duality between $\sigma_{p}$ and $\hat{\sigma}_{q}$. Since the map $\Phi$ is built using a mixture of $\sigma_{p}$ 's and $\hat{\sigma}_{q}$ 's, its diagram is a combination of the above diagrams. 

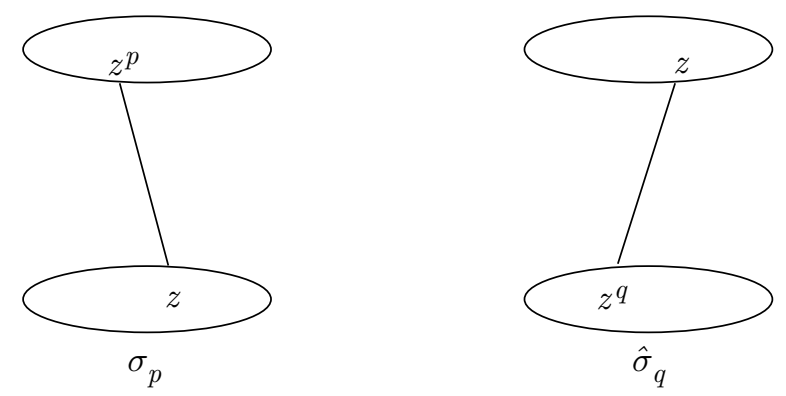

FigURE 2

Example. The embedding

$$
\begin{gathered}
\Phi: C(\mathbf{T}) \oplus C(\mathbf{T}) \rightarrow C(\mathbf{T}) \otimes \mathbf{M}_{6} \oplus C(\mathbf{T}) \otimes \mathbf{M}_{6}, \\
\Phi(f \oplus g)=\left(\begin{array}{ccc}
\hat{\sigma}_{-2}(f) & 0 & 0 \\
0 & \sigma_{-1}(f) & 0 \\
0 & 0 & \hat{\sigma}_{3}(g)
\end{array}\right) \oplus\left(\begin{array}{cccc}
\sigma_{1}(f) & 0 & 0 & 0 \\
0 & \sigma_{-3}(f) & 0 & 0 \\
0 & 0 & \hat{\sigma}_{-3}(g) & 0 \\
0 & 0 & 0 & \sigma_{-2}(g)
\end{array}\right)
\end{gathered}
$$

has the diagram in Figure 3.

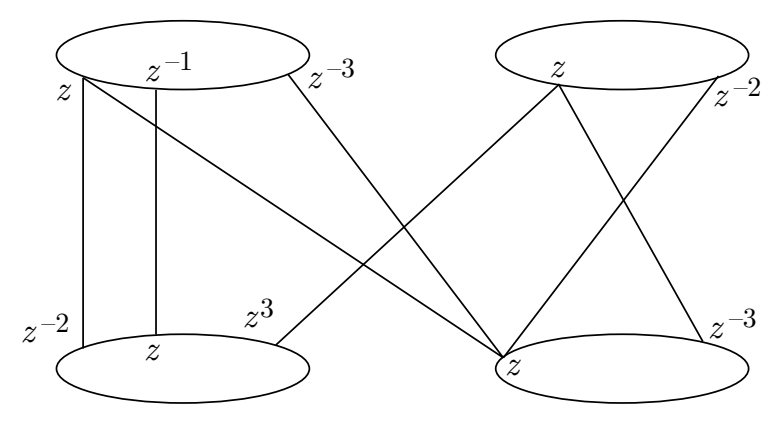

FiguRE 3

The edges in the diagram of $\Phi$ are of the form $\left(z, z^{p}\right)$ or $\left(z^{q}, z\right)$, for some non-zero $p$ and $q$. (We may have multiplicity, but we prefer not to complicate the notation.)

If we repeat the diagram of $A$ infinitely many times, we get a Bratteli diagram which corresponds to an AF-algebra. We do the same thing with the diagram of $\Phi$, and we get an infinite circle diagram, corresponding to an AT-algebra (see [De2]). This diagram carries a vertical symmetry, and we can define a shift on the space of infinite paths.

More precisely, let $E$ denote the space of edges in the diagram of $\Phi$, endowed with the product topology from the cartesian product of the vertex space $\mathbf{T} \sqcup \ldots \sqcup \mathbf{T}$ ( $N$ times) with itself, and let

$$
X:=\left\{\gamma \in \prod_{1}^{\infty} E \mid \operatorname{end}\left(\gamma_{n}\right)=\operatorname{start}\left(\gamma_{n+1}\right)\right\}
$$


be the space of infinite paths with the product topology. The elements $\gamma$ of $X$ are concatenations of edges $\gamma_{1}, \gamma_{2}, \ldots$. Since $E$ is a compact space (topologically, it is a disjoint union of circles), $X$ is also compact. Define $\sigma: X \rightarrow X$,

$$
\sigma\left(\gamma_{1} \gamma_{2} \ldots\right)=\gamma_{2} \gamma_{3} \ldots
$$

This is a continuous, surjective map and, taking into account the form of the edges, it is a local homeomorphism. We define the analogue of the Cuntz-Krieger groupoid to be

$$
\Gamma:=\left\{(\xi, k, \eta) \in X \times \mathbf{Z} \times X \mid \xi_{n}=\eta_{n+k} \text { for large } n\right\} .
$$

The unit space of $\Gamma$ may be identified with $X$. The range and source maps are $r, s: \Gamma \rightarrow X$,

$$
r(\xi, k, \eta)=\xi, \quad s(\xi, k, \eta)=\eta
$$

The multiplication is given by

$$
(\xi, k, \eta)(\eta, l, \zeta)=(\xi, k+l, \zeta)
$$

and the inverse of $(\xi, k, \eta)$ is $(\eta,-k, \xi)$. The topology of $\Gamma$ is described in the following way: a basis of open sets is defined by

$$
Z(\alpha, U, \beta, V):=\left\{(\xi, k, \eta) \in \Gamma|k=| \beta|-| \alpha \mid, \quad \xi_{1} \ldots \xi_{|\alpha|} \in U, \quad \eta_{1} \ldots \eta_{|\beta|} \in V\right\},
$$

where $\alpha \in \prod_{1}^{|\alpha|} E, \beta \in \prod_{1}^{|\beta|} E$ are finite paths of lengths $|\alpha|,|\beta|$, and $U, V$ are open sets containing $\alpha$ and $\beta$, respectively. With this topology, $\Gamma$ becomes a locally compact (Hausdorff) groupoid. Since a finite number of edges end in each vertex, and this number is the same for each connected component, the counting measures will form a Haar system for $\Gamma$. Therefore we may consider the $\mathbf{C}^{*}$-algebra of the groupoid $\Gamma$.

Definition. The generalized Cuntz-Krieger algebra $\mathcal{O}_{\Phi}$ is defined to be the $\mathbf{C}^{*}$ algebra of the groupoid $\Gamma$.

\section{Structure and K-theory of $\mathcal{O}_{\Phi}$}

In order to compute the K-theory of $\mathcal{O}_{\Phi}$, we will write it as a crossed product of an inductive limit of circle algebras by a corner endomorphism, and we will use a variant of the Pimsner-Voiculescu exact sequence obtained by Paschke. First, we will define some equivalence relations. Let

$$
\begin{gathered}
R^{k}:=\left\{(\xi, \eta) \in X \times X \mid \xi_{n}=\eta_{n} \forall n>k\right\}, \\
R^{\infty}:=\bigcup_{k=0}^{\infty} R^{k} .
\end{gathered}
$$

Then $R^{\infty}$ is a subgroupoid of $\Gamma$, with the same unit space. In the sequel we will suppose that each orbit with respect to $R^{\infty}$ is dense. We will say in this case that $\sigma$ is minimal. This hypothesis will ensure that the $\mathbf{C}^{*}$-algebra $\mathbf{C}^{*}\left(R^{\infty}\right)$ is simple.

Recall that in the definition of $\Phi$, the integer $p_{k}$ is the number of edges ending on the $k$-th circle of the vertex space. Note that this number will be the same for each level of the infinite diagram. The shift $\sigma$ induces a ${ }^{*}$-endomorphism of $\mathbf{C}^{*}\left(R^{\infty}\right)$, denoted $\sigma^{\#}$, via the formula

$$
\left(\sigma^{\#} f\right)(\xi, \eta)=\frac{1}{\sqrt{p_{k} p_{l}}} f(\sigma \xi, \sigma \eta)
$$


for $f \in C_{c}\left(R^{\infty}\right)$, where the edge $\xi_{1}$ ends on the $k$-th circle, and the edge $\eta_{1}$ ends on the $l$-th circle. Moreover, $\sigma^{\#}$ is induced by a non-unitary isometry $v$, in the sense that

$$
\sigma^{\#} f=v * f * v^{*},
$$

where

$$
v(\xi, n, \eta)= \begin{cases}1 / \sqrt{p_{k}}, & \text { if } n=-1, \eta=\sigma \xi, \\ 0, & \text { otherwise }\end{cases}
$$

Indeed,

$$
\begin{array}{rl}
v * f & * v^{*}(\xi, \eta)=\sum_{\gamma} \sum_{i}(v * f)(\xi,-i, \gamma) v^{*}(\gamma, i, \eta) \\
& =(v * f)(\xi,-1, \sigma \eta) v^{*}(\sigma \eta, 1, \eta)=\frac{1}{\sqrt{p_{l}}} \sum_{\beta} \sum_{j} v(\xi,-1-j, \beta) f(\beta, j, \sigma \eta) \\
& =\frac{1}{\sqrt{p_{l}}} v(\xi,-1, \sigma \xi) f(\sigma \xi, 0, \sigma \eta)=\frac{1}{\sqrt{p_{k} p_{l}}} f(\sigma \xi, \sigma \eta)=\sigma^{\#} f(\xi, \eta) .
\end{array}
$$

It is easy to check that

$$
\left(v^{*} * v\right)(\xi, n, \eta)= \begin{cases}1, & \text { if } \xi=\eta \text { and } n=0, \\ 0, & \text { otherwise }\end{cases}
$$

and

$$
\left(v * v^{*}\right)(\xi, n, \eta)= \begin{cases}1 / p_{k}, & \text { if } \sigma \xi=\sigma \eta \text { starts on the } k \text {-th circle and } n=0, \\ 0, & \text { otherwise. }\end{cases}
$$

Note that $\sigma^{\#}(1)=v * v^{*}$ is a proper projection. It follows that

$$
\mathcal{O}_{\Phi}=\mathbf{C}^{*}\left(\mathbf{C}^{*}\left(R^{\infty}\right), v\right)=\mathbf{C}^{*}\left(R^{\infty}\right) \times_{\sigma^{\#}} \mathbf{Z}_{+}
$$

in the sense of Paschke [Pa1], [Pa2]. As in [De1], the following result is crucial.

Theorem. Suppose that the shift $\sigma: X \rightarrow X$ is minimal. Then we have an exact sequence

$$
\begin{aligned}
& K_{0}\left(\mathbf{C}^{*}\left(R^{\infty}\right)\right) \stackrel{i d-\left[\sigma^{\#}\right]_{0}}{\longrightarrow} K_{0}\left(\mathbf{C}^{*}\left(R^{\infty}\right)\right) \stackrel{[i]_{0}}{\longrightarrow} K_{0}\left(\mathcal{O}_{\Phi}\right) \\
& \partial_{1} \uparrow \quad \downarrow \partial_{0} \\
& K_{1}\left(\mathcal{O}_{\Phi}\right) \stackrel{[i]_{1}}{\longleftarrow} K_{1}\left(\mathbf{C}^{*}\left(R^{\infty}\right)\right) \stackrel{i d-\left[\sigma^{\#}\right]_{1}}{\longleftarrow} K_{1}\left(\mathbf{C}^{*}\left(R^{\infty}\right)\right)
\end{aligned}
$$

where $i: \mathbf{C}^{*}\left(R^{\infty}\right) \rightarrow \mathcal{O}_{\Phi}$ is the inclusion map and id is the identity on K-theory.

Proof. Using the fact that $\sigma^{\#} f=v * f * v^{*}$, it follows that $\sigma^{\#}\left(\mathbf{C}^{*}\left(R^{\infty}\right)\right)$ is a corner in $\mathbf{C}^{*}\left(R^{\infty}\right)$. Since $\mathbf{C}^{*}\left(R^{\infty}\right)$ is simple, it is a full corner and we apply Theorem 4.1 of $[\mathrm{Pa} 2]$.

Therefore, in order to compute $K_{*}\left(\mathcal{O}_{\Phi}\right)$, we need to know $K_{*}\left(\mathbf{C}^{*}\left(R^{\infty}\right)\right)$ and the maps $\left[\sigma^{\#}\right]_{*}$. For the computation of the $K$-theory, it is convenient to think of $\mathbf{C}^{*}\left(R^{\infty}\right)$ as an inductive limit of circle algebras. If we denote by $X_{n}$, for $n \geq 1$, the set of finite paths of length $n$, and by $X_{0}$ the vertex space $\mathbf{T} \sqcup \ldots \sqcup \mathbf{T}$, we have natural maps $r_{n}: X \rightarrow X_{n}, n \geq 0$. Moreover,

$$
X=\lim _{\longleftarrow} X_{n} .
$$


Consider the r-discrete equivalence relations $R_{0}=$ diagonal of $X_{0} \times X_{0}$, and for $n \geq 1$,

$$
R_{n}:=\left\{\left(\xi_{1} \ldots \xi_{n}, \eta_{1} \ldots \eta_{n}\right) \in X_{n} \times X_{n} \mid \operatorname{end}\left(\xi_{n}\right)=\operatorname{end}\left(\eta_{n}\right)\right\} .
$$

Then $\mathbf{C}^{*}\left(R_{n}\right)$ is a circle algebra, and the inclusion $\mathbf{C}^{*}\left(R_{0}\right) \rightarrow \mathbf{C}^{*}\left(R_{1}\right)$ is exactly $\Phi$. Moreover,

$$
\mathbf{C}^{*}\left(R^{\infty}\right) \simeq \underset{\lim }{\longrightarrow} \mathbf{C}^{*}\left(R_{n}\right)
$$

(see [De2]). Note that the maps $r_{n}$ induce inclusions

$$
\mathbf{C}^{*}\left(R_{n}\right) \stackrel{r_{n}^{*}}{\rightarrow} \mathbf{C}^{*}\left(R^{n}\right), \quad\left(r_{n}^{*} f\right)(\xi, \eta)=f\left(\xi_{1} \ldots \xi_{n}, \eta_{1} \ldots \eta_{n}\right) .
$$

Since $\sigma^{\#}\left(\mathbf{C}^{*}\left(R^{n}\right)\right) \subset \mathbf{C}^{*}\left(R^{n+1}\right)$, we get by restriction a map

$$
\begin{aligned}
\sigma^{\#}: & \mathbf{C}^{*}\left(R_{n}\right) \rightarrow \mathbf{C}^{*}\left(R_{n+1}\right), \\
& \left(\sigma^{\#} f\right)\left(\xi_{1} \ldots \xi_{n+1}, \eta_{1} \ldots \eta_{n+1}\right)=\frac{1}{\sqrt{p_{k} p_{l}}} f\left(\xi_{2} \ldots \xi_{n+1}, \eta_{2} \ldots \eta_{n+1}\right),
\end{aligned}
$$

if the edges $\xi_{1}, \eta_{1}$ end on the $k$-th and $l$-th circle, respectively. In order to determine $\left[\sigma^{\#}\right]_{*}: K_{*}\left(\mathbf{C}^{*}\left(R^{\infty}\right)\right) \rightarrow K_{*}\left(\mathbf{C}\left(R^{\infty}\right)\right)$, we have to compute the maps induced at $K$ theory by

$$
\sigma^{\#}: \mathbf{C}^{*}\left(R_{0}\right) \rightarrow \mathbf{C}^{*}\left(R_{1}\right)
$$

Denote by $e_{1}, \ldots, e_{N}$ the characteristic functions of the circles in the vertex space, and let $u_{1}, \ldots, u_{n}$ be the functions $u_{j}(z)=z, j=1, \ldots, N$. Then $\sigma^{\#}\left(e_{j}\right)$ is the projection in $C(\mathbf{T}) \otimes \mathbf{M}_{p_{j}}$ given by the matrix with all entries equal to $1 / p_{j}$, and $\sigma^{\#}\left(e_{j} \otimes u_{j}\right)$ is equal to the above projection tensored with $u_{j}$. It follows that $\sigma^{\#}: \mathbf{C}^{*}\left(R_{0}\right) \rightarrow \mathbf{C}^{*}\left(R_{1}\right)$ induces the identity at the level of $K_{0}$ and $K_{1}$-theory. Since $\mathbf{C}^{*}\left(R^{\infty}\right)=\lim \mathbf{C}^{*}\left(R_{n}\right)$ is a stationary inductive limit and the maps induced at $K$-theory by the inclusions $\mathbf{C}^{*}\left(R_{n}\right) \rightarrow \mathbf{C}^{*}\left(R_{n+1}\right)$ are precisely the maps induced by $\Phi$, we have

$$
K_{*}\left(\mathbf{C}^{*}\left(R^{\infty}\right)\right)=\underset{\lim }{\longrightarrow}\left(\mathbf{Z}^{N}, \Phi_{*}\right) .
$$

We represent the inductive $\operatorname{limit} \lim \left(\mathbf{Z}^{N}, \Phi_{*}\right)$ as the set of equivalence classes of sequences $\left(x_{n}\right)_{n \in \mathbf{N}}$, where $x_{n} \in \overrightarrow{\mathbf{Z}^{N}}$ and $x_{n+1}=\Phi_{*} x_{n}$ for $n$ large, where two sequences are identified if they differ only at a finite number of components. The map $\left[\sigma^{\#}\right]_{*}: K_{*}\left(\mathbf{C}^{*}\left(R^{\infty}\right)\right) \rightarrow K_{*}\left(\mathbf{C}^{*}\left(R^{\infty}\right)\right)$ could be described by the diagram

$$
\begin{gathered}
\mathbf{Z}^{N} \stackrel{\Phi_{*}}{\longrightarrow} \mathbf{Z}^{N} \stackrel{\Phi_{*}}{\longrightarrow} \mathbf{Z}^{N} \stackrel{\Phi_{*}}{\longrightarrow} \ldots \\
i d \downarrow \quad i d \downarrow \\
\mathbf{Z}^{N \stackrel{\Phi_{*}}{\longrightarrow}} \mathbf{Z}^{N} \stackrel{\Phi_{*}}{\longrightarrow} \mathbf{Z}^{N} \stackrel{\Phi_{*}}{\longrightarrow} \ldots
\end{gathered}
$$

But $\left[\sigma^{\#}\right]_{*}$ is an isomorphism, with inverse given by

$$
\begin{aligned}
& \mathbf{Z}^{N} \stackrel{\Phi_{*}}{\longrightarrow} \mathbf{Z}^{N} \stackrel{\Phi_{*}}{\longrightarrow} \mathbf{Z}^{N} \stackrel{\Phi_{*}}{\longrightarrow} \ldots \\
& i d \downarrow \quad i d \downarrow \\
& \mathbf{Z}^{N} \stackrel{\Phi_{*}}{\longrightarrow} \mathbf{Z}^{N} \stackrel{\Phi_{*}}{\longrightarrow} \mathbf{Z}^{N} \stackrel{\Phi_{*}}{\longrightarrow} \ldots
\end{aligned}
$$


We will use the same method that Cuntz used for the computation of the $K$-theory of $\mathcal{O}_{A}$ (see $\left.[\mathrm{Cu}]\right)$. The fact is that in the six term exact sequence, we may replace the maps $\left[\sigma^{\#}\right]_{*}$ by their inverses, and we still have an exact sequence.

Main Result. Let

$$
\Phi: \bigoplus_{k=1}^{N} C(\mathbf{T}) \rightarrow \bigoplus_{k=1}^{N} C(\mathbf{T}) \otimes \mathbf{M}_{p_{k}}
$$

be an embedding of circle algebras as above, built using $\sigma_{p}$ 's and $\hat{\sigma}_{q}$ 's, and suppose that the induced shift $\sigma$ is minimal. Let $\Phi_{*}=K_{*}(\Phi): \mathbf{Z}^{N} \rightarrow \mathbf{Z}^{N}$ be the matrices induced by $\Phi$ at the level of $K$-theory. Then

$$
\begin{aligned}
& K_{0}\left(\mathcal{O}_{\Phi}\right) \simeq \operatorname{ker}\left(i d-\Phi_{1}\right) \oplus\left(\mathbf{Z}^{N} /\left(i d-\Phi_{0}\right) \mathbf{Z}^{N}\right), \\
& K_{1}\left(\mathcal{O}_{\Phi}\right)=\operatorname{ker}\left(i d-\Phi_{0}\right) \oplus\left(\mathbf{Z}^{N} /\left(i d-\Phi_{1}\right) \mathbf{Z}^{N}\right) .
\end{aligned}
$$

For the proof, we first need a lemma due to Cuntz.

Lemma (Cuntz). Consider the homomorphism

$$
j: \mathbf{Z}^{N} \rightarrow \underset{\lim }{\longrightarrow}\left(\mathbf{Z}^{N}, \Phi_{*}\right)
$$

given by

$$
x \mapsto\left(x, \Phi_{*} x, \Phi_{*}^{2} x, \ldots\right),
$$

induced by the map $K_{0}\left(\mathbf{C}^{*}\left(R_{0}\right)\right) \rightarrow K_{0}\left(\mathbf{C}^{*}\left(R^{\infty}\right)\right)$. In the limit $\lim \left(\mathbf{Z}^{N}, \Phi_{*}\right)$, every element is equivalent modulo $\left(i d-\left[\sigma^{\#}\right]_{*}^{-1}\right) \underline{\lim }\left(\mathbf{Z}^{N}, \Phi_{*}\right)$ to an element in $j\left(\mathbf{Z}^{N}\right)$. Moreover, the diagram

$$
\begin{array}{ccc}
\mathbf{Z}^{N} & \stackrel{i d-\Phi_{*}}{\longrightarrow} & \mathbf{Z}^{N} \\
j \downarrow & \downarrow j \\
\stackrel{\lim }{\longrightarrow}\left(\mathbf{Z}^{N}, \Phi_{*}\right) \stackrel{i d-\left[\sigma^{\#}\right]_{*}^{-1}}{\longrightarrow} \stackrel{\lim \left(\mathbf{Z}^{N}, \Phi_{*}\right)}{\longrightarrow}
\end{array}
$$

is commutative.

Proof of the Main Result. If $j(x) \in\left(i d-\left[\sigma^{\#}\right]_{*}^{-1}\right) \lim \left(\mathbf{Z}^{N}, \Phi_{*}\right)$, it follows that $\Phi_{*}^{k} x$ $=\left(i d-\Phi_{*}\right) y$ for some $y \in \mathbf{Z}^{N}$ and some $k \geq 0$, so that $x \in\left(i d-\Phi_{*}\right) \mathbf{Z}^{N}$. Therefore

$$
K_{*}\left(\mathbf{C}^{*}\left(R^{\infty}\right)\right) /\left(i d-\left[\sigma^{\#}\right]_{*}^{-1}\right) K_{0}\left(\mathbf{C}^{*}\left(R^{\infty}\right)\right) \simeq \mathbf{Z}^{N} /\left(i d-\Phi_{*}\right) \mathbf{Z}^{N} .
$$

If $\left(i d-\left[\sigma^{\#}\right]_{*}^{-1}\right) x=0$ for $x \in \underline{\lim }\left(\mathbf{Z}^{N}, \Phi_{*}\right)$, then $x$ is represented by a constant sequence $\left(x^{\prime}, x^{\prime}, \ldots\right), x^{\prime} \in \operatorname{ker}\left(i d \overrightarrow{-} \Phi_{*}\right)$. Therefore,

$$
\operatorname{ker}\left(i d-\left[\sigma^{\#}\right]_{*}^{-1}\right)=\operatorname{ker}\left(i d-\Phi_{*}\right) .
$$

From the six term exact sequence we get the exact sequences

$$
0 \rightarrow k e r \partial_{*} \rightarrow K_{*}\left(\mathcal{O}_{\Phi}\right) \rightarrow i m \partial_{*} \rightarrow 0 .
$$

But ker $\partial_{*} \simeq i m[i]_{*} \simeq \mathbf{Z}^{N} /\left(i d-\Phi_{*}\right) \mathbf{Z}^{N}$. Therefore we get the exact sequences

$$
\begin{aligned}
& 0 \rightarrow \mathbf{Z}^{N} /\left(i d-\Phi_{0}\right) \mathbf{Z}^{N} \rightarrow K_{0}\left(\mathcal{O}_{\Phi}\right) \rightarrow \operatorname{ker}\left(i d-\Phi_{1}\right) \rightarrow 0, \\
& 0 \rightarrow \mathbf{Z}^{N} /\left(i d-\Phi_{1}\right) \mathbf{Z}^{N} \rightarrow K_{1}\left(\mathcal{O}_{\Phi}\right) \rightarrow \operatorname{ker}\left(i d-\Phi_{0}\right) \rightarrow 0,
\end{aligned}
$$

which split, since $\operatorname{ker}\left(i d-\Phi_{*}\right)$ is a subgroup of $\mathbf{Z}^{N}$. 
Corollary. Let $\Phi, \Psi$ be two embeddings of circle algebras as in the Main Result, and suppose that the corresponding shifts are minimal. If the maps $\Phi$ and $\Psi$ induce the same maps at the level of $K$-theory, then the algebras $\mathcal{O}_{\Phi}$ and $\mathcal{O}_{\Psi}$ are isomorphic.

Proof. In this case, we can apply the result of Rørdam (see $[\mathrm{R} \varnothing]$ ), since the algebras $\mathcal{O}_{\Phi}$ and $\mathcal{O}_{\Psi}$ are classified by their K-theory.

Remark. It is natural to ask about conditions on the embedding $\Phi$ which will ensure the minimality of the shift $\sigma$. A sufficient condition is the following: there exists a positive integer $n$ such that all the entries in the matrix $\left(\Phi_{0}\right)^{n}$ are strictly larger than the corresponding entries in the matrix $\left(\hat{\Phi}_{0}\right)^{n}$, where $\hat{\Phi}$ denotes the dual of $\Phi$ (see [De2]). This is because the maps $\hat{\sigma}_{p}$ shrink the spectral variation, while the maps $\sigma_{q}$ expand it, and we want the spectral variation of each partial embedding to become arbitrary small, after taking sufficiently many iterations. In this case, it is easy to see that the AT-algebra $\mathbf{C}^{*}\left(R^{\infty}\right)$ has no proper ideal; therefore $\sigma$ is minimal. Also, it has real rank zero (loc. cit.).

Example. For the embedding $\Phi$ with the diagram given in Figure 3, we have

$$
\Phi_{0}=\left[\begin{array}{ll}
3 & 3 \\
2 & 4
\end{array}\right], \quad \Phi_{1}=\left[\begin{array}{rr}
-2 & 1 \\
-2 & -3
\end{array}\right] .
$$

The K-theory of the corresponding algebra $\mathcal{O}_{\Phi}$ is

$$
K_{0} \simeq \mathbf{Z} \oplus \mathbf{Z}_{2}, \quad K_{1} \simeq \mathbf{Z} \oplus \mathbf{Z}_{14} .
$$

\section{Comments And PRoblems}

Note that the $K_{1}$-theory of the algebras $\mathcal{O}_{\Phi}$ has torsion, so that they are true generalizations of the algebras $\mathcal{O}_{A}$. The groupoid approach has the advantage of emphasizing the triple

$$
C(X) \subset \mathbf{C}^{*}\left(R^{\infty}\right) \subset \mathcal{O}_{\Phi},
$$

and suggesting possible results about the state space of $\mathcal{O}_{\Phi}$, similar to those for the $\operatorname{algebras} \mathcal{O}_{A}$.

It should be mentioned that, following Renault's analysis of the Cuntz groupoid, one can see that our groupoid $\Gamma$ is equivalent, in the sense of Muhly, Renault and Williams, to a groupoid $\tilde{\Gamma}$ on the space $\tilde{X}$ of two-sided infinite paths. The groupoid $\tilde{\Gamma}$ is a semi-direct product of an equivalence relation and a bilateral shift. The locally compact space which realizes the $\Gamma-\tilde{\Gamma}$ equivalence is

$$
Z=\left\{(\xi, n, \tilde{\eta}) \in X \times \mathbf{Z} \times \tilde{X} \mid \xi_{k}=\tilde{\eta}_{k+n} \text { for large } k\right\} .
$$

The exact sequence of Paschke and the fact that the maps $\left[\sigma^{\#}\right]_{*}$ are isomorphisms allowed us to obtain a shortcut to the computation of K-theory.

Also, there seems to be a strong connection between the algebra $\mathcal{O}_{\Phi}$ and the Cuntz-Krieger algebra $\mathcal{O}_{A}$, where $A$ is the matrix induced by $\Phi$ at the level of $K_{0^{-}}$ theory. In terms of diagrams, one may think that we shrink each circle to a point, collapsing the corresponding edges, and we obtain a stationary Bratteli diagram for an AF-algebra. Of course, the information given by the maps $K_{1}(\Phi)$ is lost in this case.

The Markov shift $\sigma$ on the not necessarily totally disconected path space $X$ seems to be interesting in its own right. It would be desirable to compute its topological entropy in terms of the matrices induced by $\Phi$ at the level of K-theory. 
It is expected that the $\mathrm{K}$-groups of $\mathcal{O}_{\Phi}$ will have an interesting significance in ergodic theory, similar to the way the K-groups of $\mathcal{O}_{A}$ serve as invariants for the flow equivalence of $A$, a fact discovered by Bowen and Franks.

It is known (see [MRS]) that the Doplicher-Roberts algebras associated to representations of finite groups can be realized as full corners in the algebras $\mathcal{O}_{A}$ for an appropriate matrix $A$. We intend to seek 'continuous' versions of the DoplicherRoberts algebras and to see if they are related to some (group?) representations.

\section{REFERENCES}

[Bl] B. Blackadar, Symmetries of the CAR algebra, Annals of Math. 131(1990) 589-623. MR 91i: 46084

[Br1] O.Bratteli, Inductive limits of finite dimensional $C^{*}$-algebras, Trans. of the AMS 171 (1972) 195-234. MR 47:844

[Cu] J.Cuntz, A Class of $\mathbf{C}^{*}$-algebras and Topological Markov Chains II: Reducible Chains and the Ext-functor for $\mathbf{C}^{*}$-algebras, Invent. Math. 63(1981) 25-40. MR 82f:46073

[Da] M.Dădârlat, On homomorphisms of certain $C^{*}$-algebras, INCREST Preprint Series No.11, 1986.

[De1] V.Deaconu, Groupoids associated with endomorphisms , Trans. of the AMS 347(1995) 17791786. MR 95h:46104

[De2] V.Deaconu, A Path Model for Circle Algebras, J. Operator Theory 34(1995), 57-89. CMP 96:04

[Ku] A.Kumjian, Preliminary algebras arising from local homeomorphisms, Math.Scand. vol 52(1983), 269-278. MR 85b:46078

[Mu] P.S.Muhly, Coordinates in Operator Algebra, to appear.

[MRS] M.H. Mann, I. Raeburn, C.E. Sutherland, Representations of compact groups, CuntzKrieger algebras, and groupoid $\mathbf{C}^{*}$-algebras, Miniconf. Probab. Anal. (Sydney, 1991), Proc. Centre Math., vol. 29, Australian Nat. Univ., Canberra, 1992, pp. 135-144. MR 93m:46082

[MRW] P.S.Muhly, J.Renault, D.P.Williams, Equivalence and isomorphism for groupoid $\mathbf{C}^{*}$ algebras, JOT 17(1987) 3-22. MR 88h:46123

[Pa1] W.L. Paschke, The crossed product of a $C^{*}$-algebra by an endomorphism, Proc. Amer. Math. Soc.80(1980), 113-118. MR 81m:46081

[Pa2] W.L. Paschke, K-theory for actions of the circle group on $C^{*}$-algebras, JOT 6 (1981), 125133. MR 82m: 46074

[Pi] M.Pimsner, A class of $C^{*}$-algebras generalizing both Cuntz-Krieger algebras and crossed products by $\mathbf{Z}$, Preprint 1993.

[Pu] I.F.Putnam, $\mathbf{C}^{*}$-algebras from Smale spaces, Canadian J. of Math. 48 (1996), No. 1, 175195.

[Re] J.Renault, A groupoid approach to $\mathbf{C}^{*}$-algebras, Springer Lecture Notes in Math., no.793, Springer-Verlag, Berlin, 1980. MR 82h:46075

[Rø] M.Rørdam, Classification of Certain Infinite Simple $C^{*}$-algebras, III, Preprint 1994

[Th] K.Thomsen, Homomorphisms between finite direct sums of circle algebras, Linear and Multilinear Algebra 32(1992) 33-50. MR 94a:46080

Department of Mathematics, University of Iowa, Iowa City, Iowa 52242

Current address: Department of Mathematics, Iowa State University, Ames, Iowa 50011

E-mail address: vdeaconu@pollux.math.iastate.edu 